\title{
Perceptual Surprise Improves Action Stopping by Nonselectively Suppressing Motor Activity via a Neural Mechanism for Motor Inhibition
}

\author{
ID Isabella C. Dutra, ${ }^{1}$ Darcy A. Waller, ${ }^{1}$ and ${ }^{\text {DJ Jan R. Wessel }}{ }^{1,2}$ \\ ${ }^{1}$ Department of Psychological and Brain Sciences, University of Iowa, Iowa City, Iowa 52245 and ${ }^{2}$ Department of Neurology, University of Iowa Hospitals \\ and Clinics, Iowa City, Iowa 52242
}

\begin{abstract}
Motor inhibition is a cognitive control ability that allows humans to stop actions rapidly even after initiation. Understanding and improving motor inhibition could benefit adaptive behavior in both health and disease. We recently found that presenting surprising, task-unrelated sounds when stopping is necessary improves the likelihood of successful stopping. In the current study, we investigated the neural underpinnings of this effect. Specifically, we tested whether surprise-related stopping improvements are due to a genuine increase in motor inhibition. In Experiment 1, we measured motor inhibition in primary motor cortex of male and female humans by quantifying corticospinal excitability (CSE) via transcranial magnetic stimulation and electromyography during a hybrid surprise-Go/ NoGo task. Consistent with prior studies of motor inhibition, successful stopping was accompanied by nonselective suppression of CSE; that is, CSE was suppressed even in task-unrelated motor effectors. Importantly, unexpected sounds significantly increased this motorsystem inhibition to a degree that was directly related to behavioral improvements in stopping. In Experiment 2, we then used scalp encephalography to investigate whether unexpected sounds increase motor-inhibition-related activity in the CNS. We used an independent stop-signal localizer task to identify a well characterized frontocentral low-frequency EEG component that indexes motor inhibition. We then investigated the activity of this component in the surprise-Go/NoGo task. Consistent with Experiment 1, this signature of motor inhibition was indeed increased when NoGo signals were followed by unexpected sounds. Together, these experiments provide converging evidence suggesting that unexpected events improve motor inhibition by automatically triggering inhibitory control.
\end{abstract}

Key words: corticospinal excitability; Go/NoGo task; motor evoked potentials; motor inhibition; stop-signal P3; surprise

Significance Statement

The ability to stop ongoing actions rapidly allows humans to adapt their behavior flexibly and rapidly. Action stopping is important in daily life (e.g., stopping to cross the street when a car approaches) and is severely impaired in many neuropsychiatric disorders. Therefore, finding ways to improve action stopping could aid adaptive behaviors in health and disease. Our current study shows that presenting unexpected sounds in stopping situations facilitates successful stopping. This improvement is specifically due to a surprise-related increase in a neural mechanism for motor inhibition, which rapidly suppresses the excitability of the motor system after unexpected events. These findings suggest a tight interaction between the neural systems for surprise processing and motor inhibition and yield a promising avenue for future research.

\section{Introduction}

Humans are able to stop already initiated actions rapidly before motor emission. This ability is key to maintaining safe goal-

\footnotetext{
Received Oct. 26, 2017; revised Dec. 27, 2017; accepted Dec. 28, 2017.

Author contributions: J.R.W. designed research;I.C.D., D.A.W., and J.R.W. performed research;I.C.D., D.A.W., and J.R.W. analyzed data; J.R.W. wrote the paper.

This work was supported by the Roy J. Carver Foundation (J.R.W.), the lowa Center for Research by Undergraduates (fellowship granted to I.D.), and the National Institutes of Health (Training Grant 1T32GM108540-01 to D.A.W.).

The authors declare no competing financial interests.

Correspondence should be addressed to Dr. Jan R. Wessel, University of lowa Hospitals and Clinics, Department of

Neurology, 200 Hawkins Drive, 1042 ML, lowa City, IA 52242. E-mail: Jan-wessel@uiowa.edu.
}

directed behavior in a rapidly changing environment, for example, when suddenly noticing an approaching vehicle while crossing the street. In the laboratory, action stopping is tested in Go/NoGo (Donders, 1969) and stop signal tasks (Logan and Cowan, 1984), which have generated detailed insights into the fronto-basal ganglia neural mechanism underlying motor inhibition (Aron et al., 2014; Jahanshahi et al., 2015; Kenemans, 2015; Duque et al., 2017). This mechanism inhibits motor activity by 
momentarily suppressing the corticospinal excitability (CSE) of targeted muscles (Coxon et al., 2006; Stinear et al., 2009; van den Wildenberg et al., 2010). When rapidly recruited, this suppression extends even beyond targeted muscles and affects the entire motor system (Badry et al., 2009; Cai et al., 2012; Majid et al., 2012; Wessel et al., 2013).

Because of the importance of stopping in everyday life, as well as the common stopping impairments found in neuropsychiatric diseases (Eagle et al., 2008; Bari and Robbins, 2013) and normal aging (Coxon et al., 2012), much research has targeted factors that can improve motor inhibition; for example, pharmacology (Eagle et al., 2008; Chamberlain et al., 2009), brain stimulation (Ray et al., 2009; Swann et al., 2011), and practice (Manuel et al., 2013; Chevalier et al., 2014). Recently, we found that presenting task-unrelated unexpected sounds when motor inhibition is needed significantly improves stopping success (Wessel, 2017a). This finding could yield insights into the mechanisms underlying action stopping and stimulus-driven attention and could also have real-world implications (e.g., for the design of human-machine interfaces such as lane change assist systems in modern cars). However, it is unclear whether this surprise-related action stopping benefit is due to a genuine improvement in motor inhibition. Unexpected perceptual events have well known effects on attention (Posner, 1980; Corbetta et al., 2008) and arousal (Preuschoff et al., 2011; Sara and Bouret, 2012) and thus could influence stopping success without actually improving motor inhibition. Therefore, the current study aimed to test whether the behavioral improvement in stopping after unexpected sounds is due to a genuine surprise-related increase in motor inhibition (Wessel and Aron, 2013).

In Experiment 1, we measured motor inhibition at the level of the motor system in healthy humans performing a Go/NoGo task. In this task (the same as in our behavioral study; Wessel, 2017a), unexpected sounds presented $50 \mathrm{~ms}$ after NoGo signals lead to increased success in stopping foot movements. We quantified CSE of a task-unrelated hand muscle using transcranial magnetic stimulation (TMS). Consistent with prior studies of action stopping, CSE was nonselectively suppressed on successfully stopped NoGo trials (i.e., stopping the foot response suppressed CSE in task-unrelated hand muscles). Importantly, when NoGo signals were accompanied by unexpected sounds, this corticomotor suppression was significantly increased.

In Experiment 2, we then measured scalp EEG during this hybrid surprise-Go/NoGo task and tested whether unexpected sounds increased motor-inhibition-related activity in the CNS. Specifically, in each subject, we used a stop signal task as an independent localizer to evoke and identify independent signal components that index the motor inhibition process. In the stop signal task, motor inhibition is indexed by frontocentral lowfrequency scalp activity that shows an earlier onset for successful versus failed stop trials within subjects (Kok et al., 2004) and correlates with the speed of stopping between subjects (Wessel and Aron, 2015). In Go/NoGo tasks, this activity is increased during successful NoGo trials (Smith et al., 2008). Additionally, its amplitude is directly related to the amount of successfully inhibited prepotent motor activity on NoGo trials (Wessel, 2017b), which further supports its role as a neural index of motor inhibition. After identifying this component for each subject from the stop signal localizer task using independent component analysis (ICA) (Jutten and Herault, 1991), we tested whether its activity in the surprise-Go/NoGo task was increased in trials with unexpected sounds.
Our results provide converging evidence for an automatic engagement of the neural system for motor inhibition after surprising events, which improves action stopping by rapidly and nonselectively suppressing corticomotor excitability.

\section{Materials and Methods \\ Experiment 1}

Participants. We conducted a power analysis to motivate our sample size a priori. To this end, we merged the behavioral data from the four experiments from our prior behavioral study (Wessel, 2017a) with the behavioral data from Experiment 2 (which was collected before Experiment 1 and analyzed in parallel) to calculate an estimate of the effect size of the behavioral action stopping benefit produced by the unexpected sounds. In this combined sample of 106 (86 participants from Wessel, 2017a; 19 participants used in Experiment 2; 1 participant who successfully completed the hybrid Go/NoGo task in Experiment 2, but not the rest of the experiments), the effect size was $d=0.83$. To achieve a power of 0.99 at a two-sided $\alpha$-level of $p=0.05$, we therefore estimated the necessary sample size as $n=16$ (effect size estimation was done using GPower 3, Faul et al., 2007; RRID:SCR_013726). Young healthy adult volunteers from the Iowa City community were recruited via a research-dedicated E-mail list, as well as through the University of Iowa Department of Psychological and Brain Sciences' online subject recruitment tool. Two datasets had to be replaced, one because of an overheating TMS coil and one because no reliable motor-evoked potential (MEP) hotspot could be found. The final sample consisted of 12 females and four males (mean age: 21.6 years, SD: 3.85), all right-handed. Participants were compensated either via course credit or an hourly payment of $\$ 15$. The procedure was approved by the University of Iowa Institutional Review Board (\#201612707).

Hybrid surprise-Go/NoGo task. Stimuli for all behavioral tasks were presented using the Psychophysics toolbox (Brainard et al., 1997; RRID:SCR_002881) under MATLAB 2015b (TheMathWorks; RRID: SCR_001622). The task was identical to Experiment 1 from our prior behavioral study (Wessel, 2017a), which in turn was adapted from a similar task (Leiva et al., 2015). The following description is adapted from the original study (Wessel, 2017a); a task diagram can be found in Figure 1. The imperative Go/NoGo stimuli were white letters "W" and "M" $(0.8 \times 0.8 \mathrm{~cm})$, which appeared against a black background $3 \mathrm{~cm}$ to the left or right of a central fixation cross. The Go/NoGo mapping to the two letters was counterbalanced. Participants responded to the stimuli by using a foot pedal (Kinesis Savant Elite 2) with their left or right foot, depending on which side the stimulus was presented (in Experiment 2, EEG, they responded by pushing the "q" or " $p$ " keys on the keyboard using their left or right index fingers). Each trial began with a fixation cross (300 ms), which was followed by the Go/NoGo stimulus. Then, 50 $\mathrm{ms}$ after the Go/NoGo stimulus, a sound was played. The standard sound was a $200 \mathrm{~ms}$ sine-wave sound at $600 \mathrm{~Hz}$ and occurred on $80 \%$ of trials and the unexpected sounds (20\% of trials) were the same birdsong segments used in a prior study (Wessel, 2018). These were 90 samples taken from European starling songbirds (courtesy of Jordan A. Comins), which were matched in amplitude envelope to the standard sine wave tone. Participants were instructed that the sound would be irrelevant to their response. One-third of the trials were NoGo trials and two-thirds were Go trials. Similar to Leiva et al. (2015), we used an adaptive deadline procedure for Go reaction times. After three correct responses, the deadline was adjusted downward by $50 \mathrm{~ms}$ and, after each miss, it was adjusted by $50 \mathrm{~ms}$ in the opposite direction. The initial deadline was $500 \mathrm{~ms}$. After each trial, participants received feedback ("correct" on correct Go responses, "incorrect" on incorrect Go responses, "too slow" when responses were made outside of the response deadline, "do not respond" on NoGo trials with responses, and "correct" on NoGo trials on which the response was successfully withheld). This feedback was displayed for $1000 \mathrm{~ms}$. Participants performed 15 blocks of 60 trials overall. The first block consisted of training and included expected sounds only. That block was excluded from further analysis. At the end of each block, we displayed feedback to the participants [mean reaction time (RT), number of incorrect responses and misses, percentage of successfully withheld 
responses]. Participants had to pause $15 \mathrm{~s}$ before they could proceed to the next block.

The same task was used in Experiment 2 (EEG), in which we presented the sounds through speakers, as done in our behavioral study (Wessel, 2017a). However, in Experiment 1 (TMS), sounds were presented through disposable in-ear headphones. This was done because of the audible "click" noise associated with the discharge of the TMS coil, which is relevant because the presentation of expected sounds immediately after unexpected sounds can reduce the effect of unexpected sounds on behavior (Parmentier, 2014). Participants performed 900 trials in total ( 15 blocks of 60 trials each) in Experiment 1. In Experiment 2 (EEG), they performed 600 trials ( 10 blocks of 60 trials), followed by 4 blocks of 60 trials in a nosound condition that served as an additional control.

EMG recording. EMG was recorded using a bipolar belly-tendon montage over the first dorsal interosseous muscle (FDI) of the right hand using adhesive electrodes (H124SG; Covidien), with a ground electrode placed over distal end of ulna. Electrodes were connected to a Grass P511 amplifier (1000 Hz sampling rate, filters: $30 \mathrm{~Hz}$ high-pass, $1000 \mathrm{~Hz}$ low-pass, $60 \mathrm{~Hz}$ notch). The amplified EMG data were sampled via a Micro 1401-3 sampler (Cambridge Electronic Design) and recorded to the disc using Signal software (Version 6; Cambridge Electronic Design).

TMS stimulation/motor evoked potentials. CSE was measured via MEPs elicited by TMS. TMS stimulation was performed with a MagStim 200-2 system with a $70 \mathrm{~mm}$ figure-eight coil. Hotspotting was performed to identify the FDI stimulation locus and correct intensity. The coil was first placed $5 \mathrm{~cm}$ lateral and $2 \mathrm{~cm}$ anterior to the vertex and repositioned to where the largest MEPs were observed consistently. Resting motor threshold (RMT) was then defined as the minimum intensity required to induce MEPs of amplitudes exceeding $0.1 \mathrm{mV}$ peak to peak in 5 of 10 consecutive probes (Rossini et al., 1994). TMS stimulation intensity was then adjusted to $115 \%$ of RMT (mean intensity: $51.8 \%$ of maximum stimulator output; range: 43-66\%) for stimulation during the experimental task. An EMG sweep was started at $100 \mathrm{~ms}$ after Go/NoGo stimulus onset to obtain a $100 \mathrm{~ms}$ baseline EMG estimate before the TMS stimulation was triggered at $200 \mathrm{~ms}$ after $\mathrm{Go} / \mathrm{NoGo}$-stimulus onset. This time point was chosen based on a pilot experiment showing that significant CSE suppression was present at that time point in this version of the Go/NoGo trials when comparing successful NoGo with Go-trials; that is, CSE was significantly lower on NoGo versus Go trials, the expected pattern based on prior studies (see Introduction). Because of the low sampling rate of the TMS stimulator due to the necessary capacitor recharge ( $\sim 1$ pulse every 3-3.5 s), we collected an MEP estimate on every other trial during the Go/NoGo task. For the purposes of normalization of the amplitudes between subjects, we collected 10 trials of resting baseline MEP amplitudes before each block and after conclusion of the experiment.

MEP analysis. The goal of this experiment was to test whether CSE suppression after NoGo stimuli was increased when the NoGo stimuli were followed by an unexpected tone. MEPs were identified from the EMG trace via in-house software developed in MATLAB. Trials were excluded if the root mean square power of the EMG trace $100 \mathrm{~ms}$ before the TMS pulse exceeded $0.01 \mathrm{mV}$ or if the MEP amplitude did not exceed $0.01 \mathrm{mV}$. MEP amplitude was quantified with a peak-to-peak rationale, measuring the difference between maximum and minimum amplitude within a time period of $10-50 \mathrm{~ms}$ after the pulse. Both automated artifact rejection and MEP amplitude quantification were checked visually for accuracy on each individual trial for every dataset by a rater who was blinded to the specific trial type. MEP amplitudes were then averaged for each condition of interest (Go with unexpected sounds, Go with expected sounds, successful NoGo with unexpected sounds, successful NoGo with expected sounds) and normalized by dividing these amplitudes by the average baseline MEP estimate across all pre-block baseline trials (for all blocks combined).

$M E P-$ behavior relationship. In addition to aiming to demonstrate an increase of CSE suppression on unexpected versus expected tones, we also aimed to relate this increase to behavior across subjects. To this end, we calculated a simple percentual measurement that quantifies the degree of added CSE suppression on successful NoGo trials with unexpected compared with successful NoGo trials with expected tones as follows:

Additional_suppression $=100 *\left(\mathrm{CSE}_{\mathrm{NoGo}, \text { expected }}\right.$

$$
\left.-\mathrm{CSE}_{\mathrm{NoG} \text {,unexpected }}\right) / \mathrm{CSE}_{\mathrm{NoGo} \text {,expected }}
$$

Larger positive values on this measurement express greater degrees of additional CSE suppression on successful NoGo trials with unexpected tones.

Experimental design and statistical analysis. All analyses were done on the sample described above, which consisted of 12 females and four males (mean age: 21.6 years, SD: 3.85 ), all right-handed. The main hypotheses were tested as follows. To investigate the behavioral effect of unexpected sounds in the hybrid surprise/NoGo task, we compared both Go trial RT and NoGo success rate (proportion of NoGo trials with successfully withheld responses relative to all NoGo trials) across both conditions (expected sound, unexpected sound, no sound) using a $t$ test at an $\alpha$ level of $p<0.05$. To investigate the predicted condition differences with regard to CSE suppression, the subject means for each condition were compared using a $2 \times 2$ repeated-measures ANOVA with the factors GO-NOGO and UNEXPECTED-EXPECTED, performed at an $\alpha$-level of $p<0.05$. 


\section{CORTICO-SPINAL EXCITABILITY OF TASK-UNRELATED FDI MUSCLE Unexpected sounds increase CSE suppression Added CSE suppression predicts Nogo success}
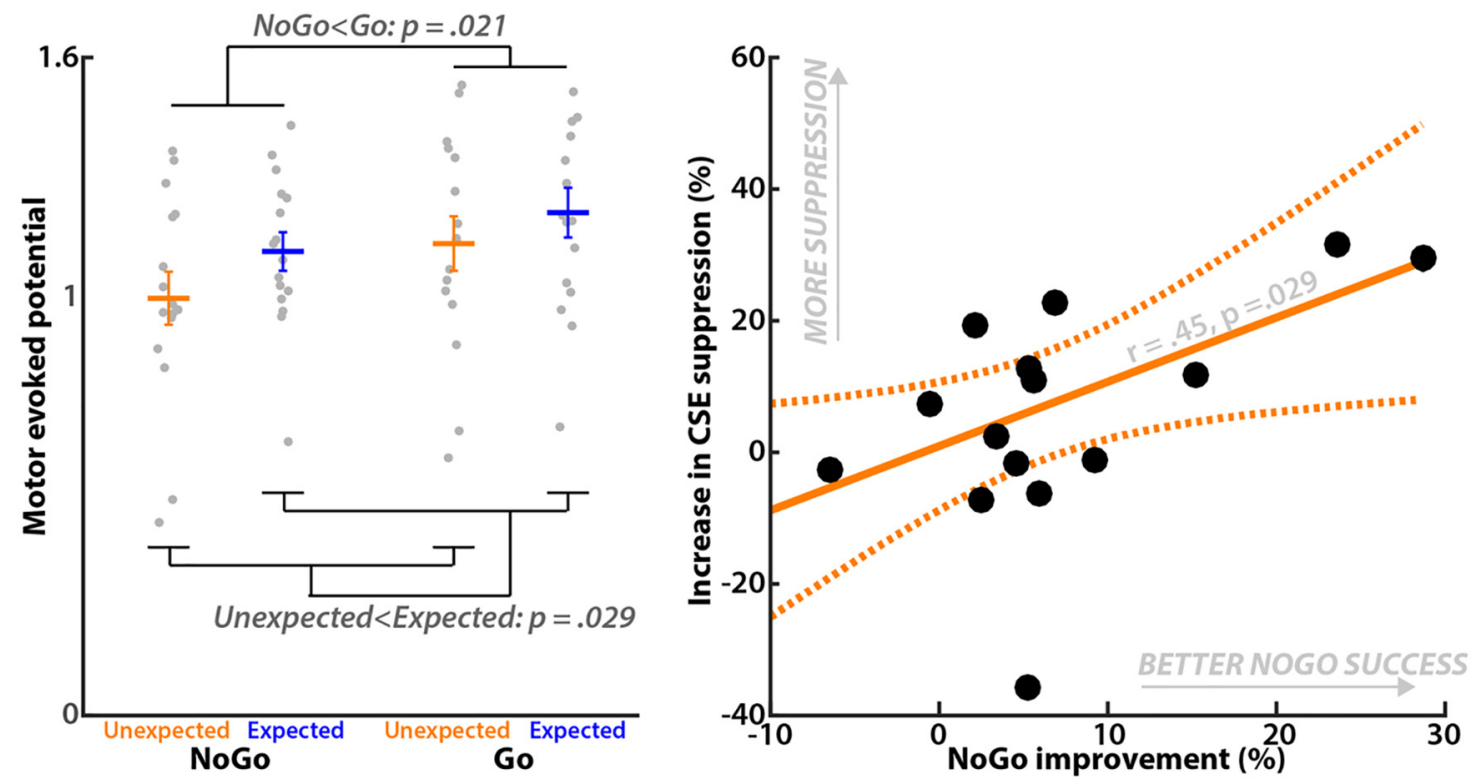

Figure 2. Corticospinal excitability in the hybrid surprise-Go/NoGo task (Experiment 1). CSE was measured at $150 \mathrm{~ms}$ after sound onset. Left plot shows the condition averages, revealing significant main effects of both $\mathrm{NoGO}_{0}<\mathrm{Go}$ and unexpected sounds $<$ expected sounds. Gray circles show individual subjects, horizontal lines denote the mean, error bars denote the SEM. Right plot shows the correlation between the degree of stopping improvement on unexpected versus expected tones ( $x$-axis) and the percentual increase in CSE suppression on successful NoGo trials with unexpected versus expected tones ( $y$-axis). Orange lines show the robust model fit between both variables, dashed line shows the confidence interval. One outlier has been removed from the graph (Cook's $d=2.25$, nonrobust model leverage: 0.42 ).

To investigate the correlation between CSE suppression and behavior, the percentual value described in the previous section was correlated to the degree of behavioral benefit on successful stopping incurred by the unexpected tones; that is, the increase in successful stopping percentage on unexpected compared with expected trials across subjects. This was done using MATLAB's fitlm() function using the robustfit algorithm (default settings).

Open data. All data and procedures for both experiments can be found on the Open Science Framework at https://osf.io/uk9jw/.

\section{Experiment 2}

Participants. Based on our prior estimation of the behavioral effects of unexpected events on NoGo success ( $d=0.81$ in a sample of $n=86$, Wessel, 2017a), we estimated that a sample size of $n=19$ would result in a power of 0.99 to detect a significant effect at a two-sided $\alpha$ level of $p<$ 0.05 . Therefore, 21 right-handed healthy young adult volunteers were recruited in the same way as described for Experiment 1. Data from two subjects had to be replaced, one because of a hardware defect and one because they opted out of the study before completing all experiments due to a lack of time. The final sample consisted of eight females and 11 males (mean age: 20.32 years, SD: 1.38). Participants were compensated for participation either via course credit or an hourly payment of $\$ 15$. The procedure was approved by the University of Iowa Institutional Review Board (\#201511709).

Stop signal localizer task. The stop signal task was identical to our prior publications (Wessel, 2018). The following description is taken from that study; a trial diagram can be found in Figure $1 A$. Trials began with a white fixation cross on a gray background ( $500 \mathrm{~ms}$ duration), followed by a white leftward or rightward arrow (go signal). Participants had to respond as fast and accurately as possible to the arrow using their left and right index finger (the respective response buttons were " $q$ " and " $p$ " on the QWERTY keyboard). On 33\% of trials, a stop signal occurred (the arrow turned from white to red) at a delay after the go stimulus (stop signal delay, SSD). The SSD, which was initially set to $200 \mathrm{~ms}$, was dynamically adjusted in $50 \mathrm{~ms}$ increments to achieve a $\mathrm{p}$ (stop) of 0.5 : after successful stops, the SSD was prolonged; after failed stops, it was shortened. This was done independently for leftward and rightward go stim- uli: SSD started at $200 \mathrm{~ms}$ for both left and right arrow trials. Then, if a stop trial with a leftward arrow led to a failed stop, the SSD for the next leftward arrow was shortened by $50 \mathrm{~ms}$, whereas the SSD for the next rightward response remained unchanged. This way, the SSD was allowed to vary independently for each arrow/response direction. Trial duration was fixed at $3000 \mathrm{~ms}$. Six blocks of 50 trials were performed (200 go, 100 stop).

Procedure. After signing written informed consent, the EEG recording was prepared and the subjects were briefed about the first task (hybrid surprise-Go/NoGo task). After performing the 600 trials of that experiment, they repeated that same task again, but with the speakers unplugged. This no sound condition served as an additional control that allowed us to compare the neural signals to the unexpected and expected sounds in the Go/NoGo task to a condition with no sounds at all. Participants practiced this task for one block of 60 trials. They then performed 10 blocks of the regular experiment followed by four blocks of the no sound condition. The no sound control experiment was always performed after the hybrid surprise-Go/NoGo task to avoid altering the processing of the unexpected nature of the sounds in that experiment (introducing any task-irrelevant sound after performing the task without sounds could have unforeseen consequences to behavior). After these two tasks, participants performed the stop signal task. After completing all three tasks, the experimenter removed the EEG cap and participants were debriefed.

Stop signal task behavior analysis. In the stop signal task, we measured Go trial RT and failed stop trial RT to test whether the predictions of the race model of the stop signal task were fulfilled by each subject's dataset (to fulfill this requirement, failed stop RT must be faster than Go trial RT; Logan and Cowan, 1984). Furthermore, we computed stop signal RT (SSRT) for the purposes of validating that our selected EEG independent signal components reflected the motor inhibition process. The onset of the activity of that component after the stop signal should correlate positively with SSRT across subjects (Wessel and Aron, 2015). SSRT was calculated using the integration method (Boehler et al., 2012; Verbruggen et al., 2013).

EEG recording. EEG was recorded using a 62-channel electrode cap connected to two BrainVision MRplus amplifiers (BrainProducts). Two addi- 
tional electrodes were placed on the left canthus (over the lateral part of the orbital bone of the left eye) and over the part of the orbital bone directly below the left eye. The ground was placed at electrode $\mathrm{Fz}$ and the reference was placed at electrode Pz. EEG was digitized at a sampling rate of $500 \mathrm{~Hz}$.

EEG preprocessing. Data were preprocessed as described in our prior studies (Wessel, 2018, 2017b) using custom routines in MATLAB. ICA was performed using functions from the EEGLAB toolbox (Delorme and Makeig, 2004; RRID:SCR_007292). After import into MAT$\mathrm{LAB}$, all datasets were merged (the stop signal dataset was appended to the datasets from the hybrid surprise-Go/NoGo task and the no sound control) and the continuous time series were filtered using symmetric 2-way leastsquares finite impulse response filters with a high pass cutoff of $0.5 \mathrm{~Hz}$ and a low-pass cutoff of $50 \mathrm{~Hz}$. The continuous time series were then inspected visually for channels with nonstereotypic artifacts, which were excluded from further processing. The remaining data were inspected visually for segments with nonstereotyped artifact activity (e.g., muscle artifacts), which were removed from further analysis of the continuous data. After artifact removal, the data were rereferenced to the common average and subjected to a temporal infomax ICA decomposition algorithm (Bell and Sejnowski, 1995), with extension to subGaussian sources (Lee et al., 1999). The resulting component matrix was screened for components representing eye movement artifacts using outlier statistics. The ICA selection was inspected visually for accuracy of the automated classification and artifact components were removed. The remaining components were subjected to further analyses.

EEG analysis: motor inhibition component selection. We selected a single independent component from each participants' ICA transformation of the entire EEG dataset. This component was selected to reflect the properties of a neural motor inhibition process in the stop signal task. This was done according to the procedure first introduced in one of our prior studies (Wessel and Aron, 2015). The overall logic of the EEG analysis is laid out in Figure 3.

The selected components show two primary features that illustrate their relationship to motor inhibition. First, their event-related potential (ERP) shows a pronounced P3 waveform after stop/NoGo-signals, the onset of which within subjects is significantly earlier for successful versus failed stop trials (a feature that reflects the race model conceptualization of the stop signal task: if the motor inhibition process starts earlier, then stopping is more likely to be successful; Logan and Cowan, 1984; Bekker et al., 2005). Second, between subjects, the onset of this stop signal P3 correlates significantly with SSRT, with subjects with slower SSRTs showing a later average ERP onset (Wessel and Aron, 2015). In addition, the phase-independent spectral perturbations of these components yield strong activations in the lower (theta/delta) frequency ranges between 2 and $8 \mathrm{~Hz}$ (Huster et al., 2013).

To identify these components automatically, we used an algorithmic procedure that is based on the COMPASS algorithm (Wessel and Ullsperger, 2011). From each individual participant's ICA, we first selected each component with a weight matrix that had its maximal weight at one of the frontocentral electrodes (FCz, Cz, C1, C2, FC1, or FC2). We then averaged those components' back-projected channel-space activity at these frontocentral electrodes within the $500 \mathrm{~ms}$ time period following the stop signal and correlated this event-related average activity to the event-related average activity of the overall EEG data (i.e., the EEG data based on the back-projection of all nonartifact ICs for that participant) in that time range. The component that showed the highest correlation with the overall ERP was selected as the motor inhibition component. In one participant, this criterion led to the selection of the wrong component upon manual inspection of the automatic algorithm (which could be attributed to an unusually late stop signal P3 waveform in this participant). Instead of rectifying the selection manually, that participant's data were removed from further analysis of the component-level activity to avoid biasing the analysis by using a subjectively informed selection of an independent component that will be used for hypothesis testing. However, retaining this participants' data with the manually selected component did not change the results qualitatively (all effects of interest in the unexpected events-Go/NoGo task remained significant). In general, a more conventional channel space analysis of the unexpected event Go/ NoGo task EEG data based on all nonartifact components yielded the same pattern of results as the analysis of the "motor inhibition" components performed here because the activity at frontocentral electrode sites in the Go/NoGo task was clearly dominated by the component reflecting the motor inhibition process. However, because we wanted to attribute our pattern of results clearly to a change of motor-inhibition-related activity obtained from an independent contrast (in fact, from an independent task), we focus on the results from that analysis.

To test whether the selected components showed the same motorinhibition-related properties described above, we detected the onset of each participant's frontocentral P3 back projection based on the selected component. Again, this was done in accordance with our prior studies (introduced in Wessel and Aron, 2015). For each subject, we selected four groups of trials: successful stop trials, failed stop trials, and a set of matched Go trials for each type of stop trial. Specifically, "matched" go trials were selected using a subset of go trials on which the SSD staircase was at the same position as the trials included in the successful/failed stop trial sample. Then, the difference between successful/failed stop trials and the respective sample of matched go trials was tested for differences from zero using sample-by-sample paired $t$ tests between the trial-to-trial amplitudes at a 2 -sided $p<0.01$ [false discovery rate (FDR) corrected using the procedure proposed by Benjamini et al., 2006]. For each sample 
MOTOR INHIBITION INDEPENDENT COMPONENT IN STOP-SIGNAL TASK

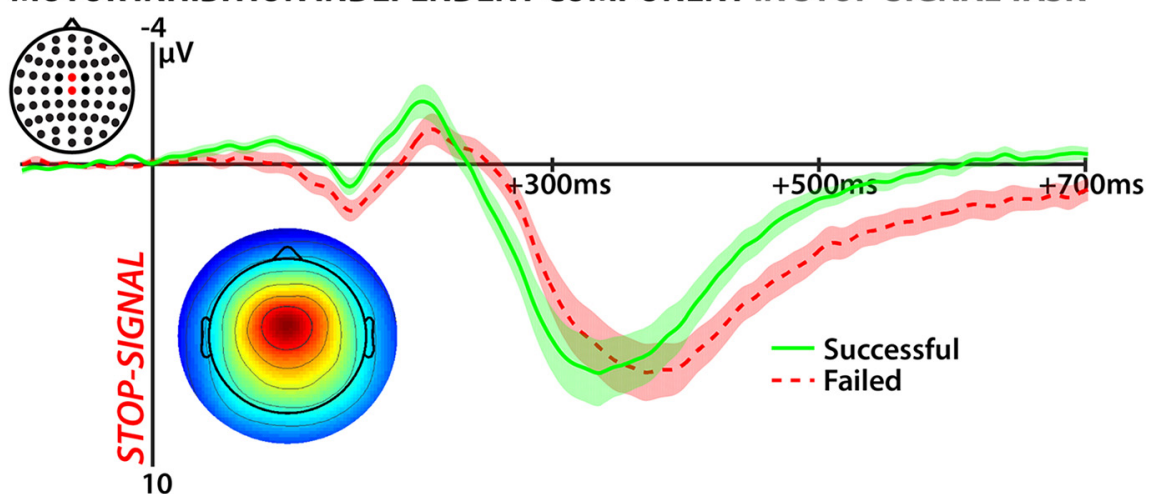

SSRT-P3 ONSET CORRELATION

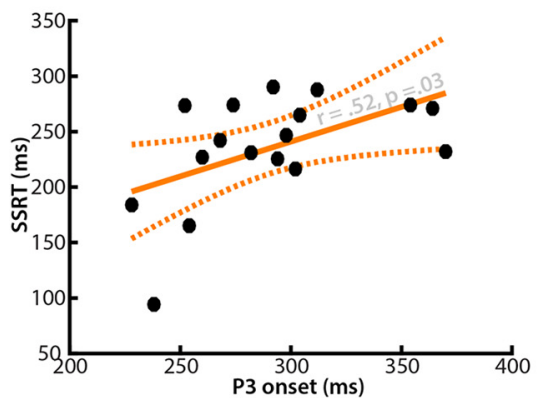

FREQUENCY PROPERTIES

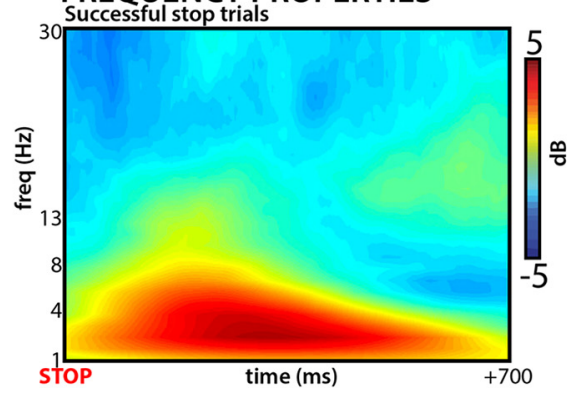

Figure 4. Stop-related properties of the selected components from the stop signal task portion of the EEG data in Experiment 2 . Top graph shows averaged back-projected stop signal ERP at channels FCz and Cz separately for successful and failed stop trials. Bottom left inlay shows the topographical representation of the average IC weight matrix (polarities rectified across subjects). Bottom left shows the correlation between the stop signal P3 onset for each subject and SSRT (integration method). Bottom right shows the time-frequency properties of the selected components on successful stop trials, showing the characteristic increase in lower-frequency bands.

point, the variance across trials (per stop/go condition) was used to construct the error term for the $t$-value. Note that this significance threshold ( $p<0.01,2$-sided) for the onset detection was significantly higher than in our initial study (Wessel and Aron, 2015; see Results for more details). These $t$ tests were performed on each sample in the time period ranging from 100 to $700 \mathrm{~ms}$ after the stop signal (with the individual time points in the stop signal waveforms being compared with matched time points in the go trial waveforms; that is, the time points in the go trial waveform at which the stop signal would have appeared according to the current SSD on that trial). This resulted in a vector of 350 logical values that showed at which sample points there was a significant difference between successful/failed stop and matched go trials. To identify the exact onset of the P3, the peak of the P3 difference wave in the critical time period (100-700 ms after the stop signal) was then detected. The $t$ test at that peak sample was significantly different from 0 in all cases; that is, all subjects showed a significant P3 for both successful and failed stop trials at least at the peak sample. Working "backwards" from that peak sample, we then identified the sample closest to the onset of the stop signal at which the positive difference wave was still significantly different from zero (at $p<0.01$, FDR corrected). Put differently, we defined the P3 onset in each participant as the time point after the stop signal at which the stretch of significant samples that included the peak of the P3 began. Again, this was done separately for successful and failed stop trials. We then compared the thus-identified single-subject onsets between successful and failed stop trials using a paired-samples $t$ test to test the relationship between stopping success and the onset timing of these components within participants. Furthermore, the onset timing of this component on successful stop trials was also correlated to SSRT to test the relationship between stopping efficacy and the onset timing of the selected P3 component between participants. This procedure is identical to our initial report of the relationship between P3 onset and SSRT/stopping success (Wessel and Aron, 2015).
Figure 4 shows the properties of the selected IC across the group and confirms that the abovementioned inhibition-related properties (earlier onset for successful vs failed stopping, high correlation of neural signal onset to SSRT, increased activity in the delta- and theta- frequency bands; more details in the Results section) that have been found for the stopping process in other studies are also present for the selected components for the current study. Our analysis of the hybrid surprise-Go/NoGo task was then based on the back projection of this (and only this) independent component into channel space. That way, the channel signal in the unexpected events Go/NoGo task was reconstructed using only the component that, in the independent stop signal task, could be identified as related to motor inhibition (for a review of this method, see Wessel, 2016). Note that, from a classic "functional localizer" perspective (as used for example in fMRI studies), one may intuitively expect that the motor inhibition IC should be generated on the stop signal portion of the data alone and then back projected onto the Go/NoGo portion of the data in attempt to avoid circularity. Although doing so would likely yield the same results, this technique would not allow the strong inference provided by the current technique: because ICA disentangles independent signal components without any knowledge of the data structure, a combined ICA provides a strong test of the hypothesis that both tasks involve the same inhibitory control process; if not, then ICA would disentangle them into separate independent components and the component selected to reflect motor inhibition in the stop signal task would show no activity differences on NoGo versus Go trials. Conversely, if the IC selected from the stop signal task portion of the data does show differences in the NoGo portion of the data, it can be concluded with some certainty that the respective underlying processes are not independent and thus likely reflect the same underlying neural generator (Onton et al., 2006; Wessel, 2016). Moreover, circularity of this analysis is prevented by selecting the component based on the stop signal trials only and then testing its activity on the Go/NoGo portion of the data.

EEG analysis: hybrid surprise-Go/NoGo-task. The primary aim of Experiment 2 was to investigate whether the presentation of unexpected sounds changed the activity of the motor inhibition components' activity to NoGo stimuli in the Go/NoGo task. To this end, we back projected the selected independent component for each subject into channel space and calculated difference waves of the back-projected ERP at frontocentral electrode channels $(\mathrm{FCz}, \mathrm{Cz})$ after successful NoGo and Go trials, separately for each sound condition (unexpected, expected, no sound). A baseline subtraction was performed before the difference wave calculation in the time range $300 \mathrm{~ms}$ before the $\mathrm{Go} / \mathrm{NoGo}$ stimulus to stimulus onset.

\section{Experimental design and statistical analysis}

All analyses were done on the sample described above, which consisted of eight females and 11 males (mean age: 20.32 years, SD: 1.38). The main hypotheses were tested as follows. To investigate the behavioral effect of unexpected sounds in the hybrid surprise/NoGo task, we compared both Go trial RT and NoGo success rate (proportion of NoGo trials with successfully withheld responses relative to all NoGo trials) across all three conditions (expected sound, unexpected sound, no sound) using a $1 \times 3$ repeated-measures ANOVA at a two-tailed $\alpha$ level of $p<0.05$. Based on the behavioral data (and corroborated by the results of Experiment 1), we predicted that unexpected sounds would amplify the inhibitory activity 
in the EEG, leading to a significant increase in the NoGo P3. To test this, we subjected each sample point in the subject average NoGo versus Go difference ERP at the frontocentral sites to a sample-to-sample $1 \times 3$ repeated-measures ANOVA with the factor SOUND (unexpected, expected, no sound). This was done for each sample ranging from 0 to 700 $\mathrm{ms}$ after stimulus. The resulting vector of $p$-values for the main effect at each sample point after the NoGo stimulus was then corrected for multiple comparisons using the FDR procedure (Benjamini et al., 2006) to a familywise error rate of $p<0.05$. The resulting effective $p$-value after FDR correction was $p=0.004$. Because we detected a significant main effect for the factor SOUND in the NoGo-P3 time range, we subjected this time range to further individual comparisons across subjects. To this end, we quantified the mean amplitude in the time range revealed by the ANOVA to yield a significant main effect separately for each trial type and subject and tested it for condition differences using $t$ tests.

\section{Results \\ Experiment 1 \\ Behavior}

We here focus on data only from the half of the trials in the experiment during which TMS stimulation occurred because those trials underlie the MEP analysis in the next section. Consistent with prior studies (Schröger, 1996; Berti and Schröger, 2004; Parmentier, 2014) and replicating our behavioral study (Wessel, 2017a), unexpected sounds led to a small, but significant slowing of RT on Go trials [417 ms (SD: 10) vs $\left.410 \mathrm{~ms}(9) ; t_{(15)}=3.02, p=0.009, d=0.2\right]$. Replicating the four experiments in our prior behavioral study, NoGo success on NoGo trials was significantly improved on trials with unexpected sounds $\left[83.8 \%\right.$ (3.9) vs $77.6 \%(4.2) ; t_{(15)}=2.58, p=$ $0.021, d=0.4]$. Therefore, whereas applying TMS reduced the size of the behavioral effect (from the initial effect size estimate of $d=0.83$ ), it was still clearly present in the data. Overall, 13 of 16 participants showed a numerical effect in the predicted direction (Fig. $1 B$, left). For the sake of completeness, the results from the non-TMS trials in this experiment showed comparable effect sizes for both the RT difference [415 $\mathrm{ms}(\mathrm{SD}: 11)$ vs $409 \mathrm{~ms}(9) ; t_{(15)}=2.34, p=0.034, d=0.18$ ] as well as the NoGo success difference between expected and unexpected tones $\left[81.2 \%(3.9)\right.$ vs $76.4 \%(3.8) ; t_{(15)}=1.8, p=$ 0.045 , one-sided, $d=0.32$ ]. However, it is worth mentioning that the unexpected absence of the sound and sensation of the TMS discharge on these trials could have influenced the results. No further analysis was performed on these trials.

\section{Motor evoked potentials}

The $2 \times 2$ ANOVA revealed significant main effects of both the factor UNEXPECTED-EXPECTED $\left(F_{(1,15)}=5.82, p=0.029\right.$, partial $\left.\eta^{2}=0.28\right)$ and the factor GO-NOGO $\left(F_{(1,15)}=6.7, p=\right.$ 0.021 , partial $\left.\eta^{2}=0.31\right)$. As can be seen from Figure 2 , the marginal means show that MEP was suppressed in the NoGo compared with the Go condition, as well as in the unexpected sound compared with the expected sound condition.

\section{MEP-behavior relationship}

In addition to the overall effect of unexpected sounds on nonselective suppression of CSE, the degree of additional CSE suppression incurred by unexpected events correlated significantly positively with the degree of behavioral improvement in stopping (standardized regression coefficient: $r=0.45, p=0.029$; Fig. 2; one outlier removed due to Cook's distance $>1$ ). Therefore, subjects in which unexpected sounds produced greater additional CSE suppression on successful stop trials also showed a greater behavioral benefit on action stopping.

\section{Experiment 2}

\section{Behavior}

As in Experiment 1, unexpected sounds led to a small, but significant slowing of RT on Go trials [353 (10.4) vs $342(9.6) ; t_{(18)}=$ $\left.5.65, p=2.33^{*} 10^{-05}, d=0.26\right]$. Also replicating Experiment 1 (and the four experiments in Wessel, 2017a), the success rate on NoGo trials (response successfully withheld) was significantly improved on trials with unexpected sounds [80\% (3.7) vs $65 \%$ $\left.(3.8) ; t_{(18)}=7.67, p=4.4^{*} 10^{-07}, d=0.95\right]$. The effect size was consistent with our prior behavioral study and larger than in Experiment 1, likely because of the absence of the sound associated with the TMS discharge. Overall, 18 of 19 participants showed a numerical effect in the predicted direction (Fig. $1 B$, right).

\section{EEG: stop signal task}

The selected motor inhibition independent components reflected the typical properties observed in prior studies of that task (see Materials and Methods). These properties of the current dataset are summarized in Figure 4. In short, the phase-locked event-related part of this component (the P3 ERP after the stop signal) showed a significantly earlier onset on successful vs failed stop trials $\left(t_{(17)}=2.035, p=0.025, d=0.49\right)$, which reflects the race model of the stop signal task (earlier stopping process onset should lead to more successful stopping). Furthermore, the onset of this stop signal P3 was significantly positively correlated with SSRT across subjects $(r=0.52, p=0.03$; one outlier was removed from the correlation due to a Cook's distance $>1$; leverage: 0.4 ). Both the size of the within-subject onset difference and the magnitude of the between-subject correlation are consistent with prior studies with larger sample sizes $(N>60$ in Wessel and Aron, 2015). However, it bears mentioning that, here, we used a much more conservative threshold for the onset detection of the P3 component (see Materials and Methods) compared with our prior studies (Wessel and Aron, 2015). Although that study used $p<0.05$ (one-sided) as an FDR-corrected onset criterion for each subject, we here used $p<0.01$ (two-sided). This was done because the quantification of the P3 onset using $p<0.05$ (onesided) in the current sample led to a positive, but highly unreliable correlation contaminated by multiple outliers, likely because of the fact that our current sample size was much smaller than the sample size of Wessel and Aron (2015). Therefore, we chose to use a more robust detection method that would identify more reliable single-subject onsets, which, however, were therefore significantly later compared with our prior study. For the sake of completeness, if the onset was quantified at a one-sided $p$ of 0.05 (as done in the original Wessel and Aron, 2015 study), the onset difference between successful and failed stop trials remained significant $\left(t_{(17)}=3.7, p=0.002, d=0.53\right)$.

Last, the event-related spectral perturbation (derived using the Hilbert transform with $1 \mathrm{~Hz}$ linear frequency spacing) shows that the vast majority of the stop-related activity increase of this component was in the low-frequency range $(2-8 \mathrm{~Hz})$, consistent with prior studies of the stop signal task (for review, see Huster et al., 2013). In summary, the selected components index motor inhibition in the stop signal task both between and within subjects.

\section{EEG: hybrid surprise-Go/NoGo task}

The sample-to-sample $1 \times 3$ ANOVA of motor-inhibitionindependent component activity at the frontocentral electrode sites $\mathrm{FCz}$ and $\mathrm{Cz}$ yielded a significant main effect of the factor SOUND on the NoGo-Go difference wave, particularly in the NoGo signal P3 time range (effective $p$-value: $p \leq 0.004$; Fig. 5). 


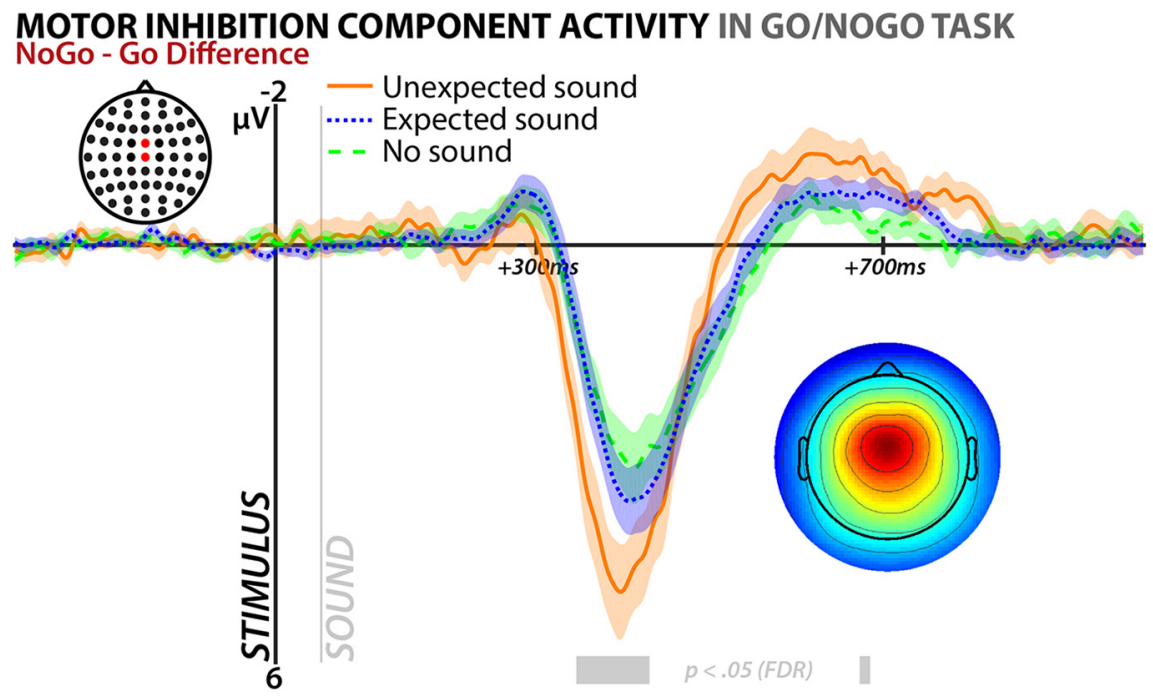

Individual condition waveforms

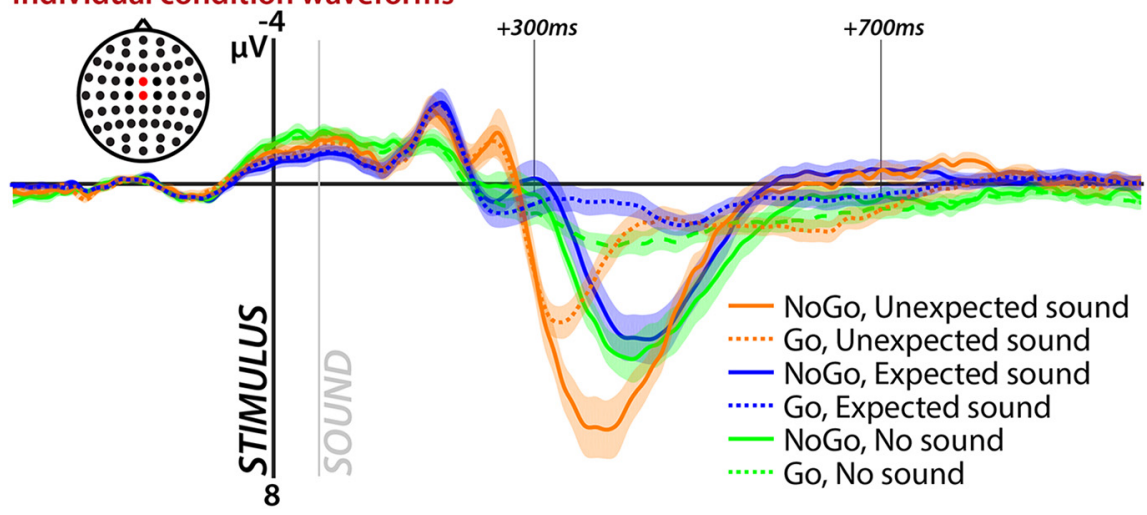

Figure 5. EEG results from Experiment 2; ERP of the motor inhibition independent component selected from the stop signal portion of the data on the surprise-Go/NoGo task portion of the data. Both graphs show average back-projected ERP at electrodes $\mathrm{FCz}$ and $\mathrm{Cz}$ time-locked to stimulus onset in the surprise-Go/NoGo task. Top plot shows difference waves between NoGo and $\mathrm{G}_{0}$ trials for all three sound conditions (unexpected, expected, no sound). Stretches of data with a significant main effect of sound condition at $p<0.05$ (FDR corrected) are highlighted in gray. Bottom plot shows individual condition waveforms.

Follow-up $t$ tests on that time range showed significant differences between the unexpected and no-sound condition $\left(t_{(17)}=\right.$ 4.21, $p=0.0006, d=0.85)$, as well as the unexpected and expected condition $\left(t_{(17)}=3.93, p=0.001, d=0.67\right)$, whereas there was no significant difference between the expected and no sound conditions $\left(t_{(17)}=0.99, p=0.34, d=0.22\right)$.

\section{Discussion}

We present data from two experiments that aimed to test whether unexpected events can benefit action stopping by invoking the activity of a neural mechanism for motor inhibition. We found that unexpected events indeed led to improvements in action stopping, which were furthermore accompanied by clear-cut motor system signatures of inhibitory control. Specifically, the nonselective CSE suppression that is commonly observed during action stopping (Badry et al., 2009; Greenhouse et al., 2012; Wessel et al., 2013; Duque et al., 2017; Wessel and Aron, 2017) was increased significantly when NoGo signals were followed by unexpected sounds. Moreover, the degree of this increase in each subject was directly related to the degree to which unexpected sounds improved action stopping. We furthermore observed that signatures of motor inhibition in the CNS (specifically, the P3 ERP that indexed the speed of motor inhibition in an independently conducted stop signal task) were increased by unexpected events as well. Therefore, we suggest that the inhibitory benefit on behavior that is produced by the unexpected events can be explained by an additional activation of a well characterized neural mechanism for motor inhibition that is automatically incurred by unexpected events.

The current study therefore supports our previously proposed hypothesis that unexpected events lead to an activation of the neural mechanism underlying motor inhibition (Wessel and Aron, 2013, 2017). This hypothesis stemmed from our observation of increased motor system suppression after unexpected events during a pure Go task (Wessel and Aron, 2013). However, that interpretation has been subject to subsequent challenges. Perhaps the key criticism was that no actual motor inhibition is necessary to explain the observed behavioral effects in that study, namely, a slowing of RTs after unexpected events. Instead, it has been asserted that these effects could be explained by a shift of attention produced by the unexpected events (Leiva et al., 2015), which would lead to slowed RTs. In this scenario, the CSE suppression would likely be an epiphenomenon. Key to this criticism is also that the task did not actually involve any outright action stopping component. However, the current study provides crucial evidence that unexpected events not only produce an actual inhibitory benefit (a behavioral improvement of action stopping), but it demonstrates direct relation of this behavioral improvement to the physiological expression of inhibition on the motor system (additional CSE suppression incurred by the unexpected events). Further support for the association between outright action stopping and unexpected events comes from Experiment 2 , in which we found that the activity of an independent neural signal component, which indexed successful stopping in an independent stop signal experiment and was significantly increased on NoGo versus Go trials in the hybrid task, was further increased when NoGo stimuli were accompanied by unexpected sounds.

Although our data provide converging evidence for the involvement of motor inhibition after unexpected events from behavior, motor systems physiology, and the CNS, they also contain a potentially interesting dissociation between central and motor system signatures of motor inhibition. Specifically, whereas the CSE data showed significant main effects of both stopping and surprise, but no significant interaction between these factors (i.e., unexpected tones suppressed CSE on Go and NoGo trials alike), the EEG data did show a significantly greater increase of inhibition-related activity on NoGo compared with Go trials (cf. the significant main effect of surprise on the NoGo-Go difference wave). Drawing conclusions from this has to be done with care because the TMS method has very limited time resolution (we only collected CSE estimates at $200 \mathrm{~ms}$ after NoGo stimuli) and it is therefore possible that the CSE suppression does show a similar time course as the EEG data. In other words, CSE suppression 
could show a relative increase on NoGo versus Go trials at later time points after the NoGo stimulus. Interestingly, the raw ERP waveforms (Fig. 5, bottom) actually suggest that the CNS activity related to motor inhibition initially shows the same properties as the CSE suppression. As can be seen, both Go and NoGo trials show an initial increase in inhibition-related activity on trials with unexpected sounds and the waveforms start to deviate only in later time periods (cf. the onset of the sample-to-sample significance of the ERP difference wave between the sound conditions; Fig. 5, top). If this pattern is veridical and reflective of a true time course of inhibition at the motor-level as well (which could be elucidated by future studies), then these data could have interesting theoretical implications for the nature of motor inhibition. Indeed, some animal work suggests that motor inhibition may occur in two stages, in which very rapid signal-related activity in subcortical inhibitory circuitry is followed by a slower inhibition process that intercepts motor emission (Schmidt et al., 2013). From this perspective, unexpected events could nondiscriminatively trigger motor inhibition early on (leading to an early uptick of inhibition-related activity regardless of whether motor inhibition is necessary or not), followed by a more graded, ongoing suppression if the Go/NoGo stimulus was successfully identified as a NoGo. However, this hypothesis is speculative and needs more investigation using specifically designed experiments.

The finding that unexpected sounds engage the same inhibitory mechanism as reactive action stopping following explicit stop signals has other highly significant implications for studies of motor inhibition. For example, a common confound in motor inhibition studies is that participants proactively engage motor inhibition when they are anticipating that they may have to stop, such as in stop signal and Go/NoGo tasks (Vink et al., 2005; Verbruggen and Logan, 2009). Several past studies found that such proactive inhibition is, at least partly, implemented by preactivating parts of the circuitry underlying reactive motor inhibition to the stop signal itself. This is especially relevant because the degree of proactive motor inhibition that is exerted by individual participants directly influences the efficiency of reactive motor inhibition and its physiological consequences on the motor level (Chikazoe et al., 2009; Greenhouse et al., 2012; Greenhouse and Wessel, 2013; Majid et al., 2013; Swann et al., 2013; Schevernels et al., 2015). Because proactive inhibition increases the efficiency of reactive inhibition, it is very helpful in real-world situations in which stopping is anticipated, such as when crossing a busy intersection with a broken traffic light. However, many real-world control scenarios that demand rapid reactive action stopping (e.g., stopping a step into the street when suddenly noticing a previously overlooked car approaching) actually may not involve significant degrees of proactive motor inhibition at all. For example, we do not usually walk into the street unless we are certain that no rapid stopping will be necessary. Therefore, classic motor inhibition paradigms such as the stop signal or Go/NoGo tasks, which do not allow the study of reactive motor inhibition in the absence of proactive motor inhibition, are of limited utility as models of many realistic stopping scenarios. However, the current findings suggest that, instead of Go/NoGo and stop signal tasks, unexpected events can be used to activate the neural circuitry underlying motor inhibition in a purely reactive, automatic fashion. Moreover, such tasks are indeed akin to the circumstances of many real-world scenarios, in which stop signals are indeed often unexpected (e.g., when we suddenly notice a previously overlooked car). In return, such real-world scenarios offer a potential explanation as to why unexpected events would activate the brain circuitry underlying motor inhibition in the first place: if many real-world situations that necessitate rapid, reactive motor inhibition are indeed caused by unexpected events, then human neural architecture would have likely evolved to be hard wired so that unexpected events automatically trigger inhibitory control.

Finally, in addition to these implications for the field of motor inhibition, our current results also have strong theoretical implications for the wider field of cognitive control. Many situations that necessitate cognitive control have behavioral effects that are similar to unexpected perceptual events. For example, cognitive control situations such as action errors, unexpected action outcomes, task-switching cues, and response conflict have all been associated with a slowing of response time (Rabbitt and Rodgers, 1977; Laming, 1979; Barceló et al., 2002; Ridderinkhof, 2002; Barceló et al., 2006; Brittain et al., 2012; Wessel et al., 2012; Cavanagh et al., 2014), suggesting that these events may trigger motor inhibition as well. Although the neural mechanisms underlying such motor slowing (as well as their functional significance) are still under ongoing debate, it would be tempting to hypothesize that motor inhibition could be a highly universal mechanism of cognitive control, which can be rapidly and reactively invoked whenever unexpected events or behavioral uncertainty occur (Cavanagh et al., 2012; Cavanagh and Shackman, 2015; Jahfari et al., 2017; Wessel and Aron, 2017).

In summary, the current study provides converging evidence from the motor system and the CNS in support of an automatic engagement of motor inhibition after unexpected perceptual events. Moreover, the degree of this engagement relates directly to the increased likelihood of successful action stopping after such events. These results suggest a tight link between the neural systems for surprise processing and motor inhibition, which has strong implications for both fields of cognitive control research. Last, these results suggest that unexpected events may be a viable model for reactive motor control in the absence of explicit stopping requirements, which can be the subject of future study.

\section{References}

Aron AR, Robbins TW, Poldrack RA (2014) Inhibition and the right inferior frontal cortex: one decade on. Trends Cogn Sci 18:177-185. CrossRef Medline

Badry R, Mima T, Aso T, Nakatsuka M, Abe M, Fathi D, Foly N, Nagiub H, Nagamine T, Fukuyama H (2009) Suppression of human corticomotoneuronal excitability during the Stop signal task. Clin Neurophysiol 120:1717-1723. CrossRef Medline

Barceló F, Periáñez JA, Knight RT (2002) Think differently: a brain orienting response to task novelty. Neuroreport 13:1887-1892. CrossRef Medline

Barceló F, Escera C, Corral MJ, Periáñez JA (2006) Task switching and novelty processing activate a common neural network for cognitive control. J Cogn Neurosci 18:1734-1748. CrossRef Medline

Bari A, Robbins TW (2013) Inhibition and impulsivity: behavioral and neural basis of response control. Prog Neurobiol 108:44-79. CrossRef Medline

Bekker EM, Kenemans JL, Hoeksma MR, Talsma D, Verbaten MN (2005) The pure electrophysiology of stopping. Int J Psychophysiol 55:191-198. CrossRef Medline

Bell AJ, Sejnowski TJ (1995) An information-maximization approach to blind separation and blind deconvolution. Neural Comput 7:1129-1159. CrossRef Medline

Benjamini Y, Krieger AM, Yekutieli D (2006) Adaptive linear step-up procedures that control the false discovery rate. Biometrika 93:491-507. CrossRef

Berti S, Schröger E (2004) Distraction effects in vision: behavioral and event-related potential indices. Neuroreport 15:665-669. CrossRef Medline

Boehler CN, Appelbaum LG, Krebs RM, Hopf JM, Woldorff MG (2012) The influence of different Stop signal response time estimation proce- 
dures on behavior-behavior and brain-behavior correlations. Behav Brain Res 229:123-130. CrossRef Medline

Brainard DH, Vision S (1997) The psychophysics toolbox. Spatial vision, 10: 433-436. CrossRef

Brittain JS, Watkins KE, Joundi RA, Ray NJ, Holland P, Green AL, Aziz TZ, Jenkinson N (2012) A role for the subthalamic nucleus in response inhibition during conflict. J Neurosci 32:13396-13401. CrossRef Medline

Cai W, Oldenkamp CL, Aron AR (2012) Stopping speech suppresses the task-irrelevant hand. Brain Lang 120:412-415. CrossRef Medline

Cavanagh JF, Shackman AJ (2015) Frontal midline theta reflects anxiety and cognitive control: meta-analytic evidence. J Physiol Paris 109:3-15. CrossRef Medline

Cavanagh JF, Figueroa CM, Cohen MX, Frank MJ (2012) Frontal theta reflects uncertainty and unexpectedness during exploration and exploitation. Cereb Cortex 22:2575-2586. CrossRef Medline

Cavanagh JF, Sanguinetti JL, Allen JJ, Sherman SJ, Frank MJ (2014) The subthalamic nucleus contributes to post-error slowing. J Cogn Neurosci 26:2637-2644. CrossRef Medline

Chamberlain SR, Hampshire A, Müller U, Rubia K, Del Campo N, Craig K, Regenthal R, Suckling J, Roiser JP, Grant JE, Bullmore ET, Robbins TW, Sahakian BJ (2009) Atomoxetine modulates right inferior frontal activation during inhibitory control: a pharmacological functional magnetic resonance imaging study. Biol Psychiatry 65:550-555. CrossRef Medline

Chevalier N, Chatham CH, Munakata Y (2014) The practice of going helps children to stop: the importance of context monitoring in inhibitory control. J Exp Psychol Gen 143:959-965. CrossRef Medline

Chikazoe J, Jimura K, Hirose S, Yamashita K, Miyashita Y, Konishi S (2009) Preparation to inhibit a response complements response inhibition during performance of a stop signal task. J Neurosci 29:15870-15877. CrossRef Medline

Corbetta M, Patel G, Shulman GL (2008) The reorienting system of the human brain: from environment to theory of mind. Neuron 58:306-324. CrossRef Medline

Coxon JP, Stinear CM, Byblow WD (2006) Intracortical inhibition during volitional inhibition of prepared action. J Neurophysiol 95:3371-3383. CrossRef Medline

Coxon JP, Van Impe A, Wenderoth N, Swinnen SP (2012) Aging and inhibitory control of action: cortico-subthalamic connection strength predicts stopping performance. J Neurosci 32:8401-8412. CrossRef Medline

Delorme A, Makeig S (2004) EEGLAB: an open source toolbox for analysis of single-trial EEG dynamics including independent component analysis. J Neurosci Methods 134:9-21. CrossRef Medline

Donders FC (1969) On the speed of mental processes. Acta Psychol 30:412431. CrossRef

Duque J, Greenhouse I, Labruna L, Ivry RB (2017) Physiological markers of motor inhibition during human behavior. Trends Neurosci 40:219-236. CrossRef Medline

Eagle DM, Bari A, Robbins TW (2008) The neuropsychopharmacology of action inhibition: cross-species translation of the stop signal and go/ no-go tasks. Psychopharmacology 199:439-456. CrossRef Medline

Faul F, Erdfelder E, Lang AG, Buchner A (2007) G*Power 3: a flexible statistical power analysis program for the social, behavioral, and biomedical sciences. Behav Res Methods 39:175-191. CrossRef Medline

Greenhouse I, Wessel JR (2013) EEG signatures associated with stopping are sensitive to preparation. Psychophysiology 50:900-908. CrossRef Medline

Greenhouse I, Oldenkamp CL, Aron AR (2012) Stopping a response has global or nonglobal effects on the motor system depending on preparation. J Neurophysiol 107:384-392. CrossRef Medline

Huster RJ, Enriquez-Geppert S, Lavallee CF, Falkenstein M, Herrmann CS (2013) Electroencephalography of response inhibition tasks: functional networks and cognitive contributions. Int J Psychophysiol 87:217-233. CrossRef Medline

Jahanshahi M, Obeso I, Rothwell JC, Obeso JA (2015) A fronto-striatosubthalamic-pallidal network for goal-directed and habitual inhibition. Nat Rev Neurosci 16:719-732. CrossRef Medline

Jahfari S, Ridderinkhof KR, Collins AGE, Knapen T, Waldorp L, Frank MJ (2017) Cross-task contributions of fronto-basal ganglia circuitry in response inhibition and conflict-induced slowing. bioRxiv:199299.

Jutten C, Herault J (1991) Blind separation of sources, part I: An adaptive algorithm based on neuromimetic architecture. Signal Process 24:1-10. CrossRef
Kenemans JL (2015) Specific proactive and generic reactive inhibition. Neurosci Biobehav Rev 56:115-126. CrossRef Medline

Kok A, Ramautar JR, De Ruiter MB, Band GP, Ridderinkhof KR (2004) ERP components associated with successful and unsuccessful stopping in a stop signal task. Psychophysiology 41:9-20. CrossRef Medline

Laming D (1979) Choice reaction performance following an error. Acta Psychol 43:199-224. CrossRef

Lee TW, Girolami M, Sejnowski TJ (1999) Independent component analysis using an extended infomax algorithm for mixed subgaussian and supergaussian sources. Neural Comput 11:417-441. CrossRef Medline

Leiva A, Parmentier FB, Elchlepp H, Verbruggen F (2015) Reorienting the mind: The impact of novel sounds on go/no-go performance. J Exp Psychol Hum Percept Perform 41:1197-1202. CrossRef Medline

Logan GD, Cowan WB (1984) On the ability to inhibit thought and action: a theory of an act of control. Psychol Rev 91:295-327. CrossRef

Majid DS Cai W, Corey-Bloom J, Aron AR (2013) Proactive selective response suppression is implemented via the basal ganglia. J Neurosci 33: 13259-13269. CrossRef Medline

Majid DS, Cai W, George JS, Verbruggen F, Aron AR (2012) Transcranial magnetic stimulation reveals dissociable mechanisms for global versus selective corticomotor suppression underlying the stopping of action. Cereb Cortex 22:363-371. CrossRef Medline

Manuel AL, Bernasconi F, Spierer L (2013) Plastic modifications within inhibitory control networks induced by practicing a stop signal task: an electrical neuroimaging study. Cortex 49:1141-1147. CrossRef Medline

Onton J, Westerfield M, Townsend J, Makeig S (2006) Imaging human EEG dynamics using independent component analysis. Neurosci Biobehav Rev 30:808-822. CrossRef Medline

Parmentier FB (2014) The cognitive determinants of behavioral distraction by deviant auditory stimuli: a review. Psychol Res 78:321-338. CrossRef Medline

Posner MI (1980) Orienting of attention. Q J Exp Psychol 32:3-25. CrossRef Medline

Preuschoff K, t'Hart BM, Einhäuser W (2011) Pupil dilation signals surprise: evidence for noradrenaline's role in decision making. Front Neurosci 5:115. CrossRef Medline

Rabbitt P, Rodgers B (1977) What does a man do after he makes an error: analysis of response programming. Q J Exp Psychol 29:727-743. CrossRef

Ray NJ, Jenkinson N, Brittain J, Holland P, Joint C, Nandi D, Bain PG, Yousif N, Green A, Stein JS, Aziz TZ (2009) The role of the subthalamic nucleus in response inhibition: Evidence from deep brain stimulation for Parkinson's disease. Neuropsychologia 47:2828-2834. CrossRef Medline

Ridderinkhof KR (2002) Micro- and macro-adjustments of task set: activation and suppression in conflict tasks. Psychol Res 66:312-323. CrossRef Medline

Rossini PM, Barker AT, Berardelli A, Caramia MD, Caruso G, Cracco RQ, Dimitrijević MR, Hallett M, Katayama Y, Lücking CH (1994) Noninvasive electrical and magnetic stimulation of the brain, spinal cord and roots: basic principles and procedures for routine clinical application. Report of an IFCN committee. Electroencephalogr Clin Neurophysiol 91:79-92. CrossRef Medline

Sara SJ, Bouret S (2012) Orienting and reorienting: the locus coeruleus mediates cognition through arousal. Neuron 76:130-141. CrossRef Medline

Schevernels H, Bombeke K, Van der Borght L, Hopf JM, Krebs RM, Boehler CN (2015) Electrophysiological evidence for the involvement of proactive and reactive control in a rewarded stop signal task. Neuroimage 121: 115-125. CrossRef Medline

Schmidt R, Leventhal DK, Mallet N, Chen F, Berke JD (2013) Canceling actions involves a race between basal ganglia pathways. Nat Neurosci 16:1118-1124. CrossRef Medline

Schröger E (1996) A neural mechanism for involuntary attention shifts to changes in auditory stimulation. J Cogn Neurosci 8:527-539. CrossRef Medline

Smith JL, Johnstone SJ, Barry RJ (2008) Movement-related potentials in the Go/NoGo task: the P3 reflects both cognitive and motor inhibition. Clin Neurophysiol 119:704-714. CrossRef Medline

Stinear CM, Coxon JP, Byblow WD (2009) Primary motor cortex and movement prevention: where Stop meets Go. Neurosci Biobehav Rev 33:662-673. CrossRef Medline

Swann NC, Tandon N, Pieters TA, Aron AR (2013) Intracranial electroencephalography reveals different temporal profiles for dorsal- and ventro- 
lateral prefrontal cortex in preparing to stop action. Cereb Cortex 23: 2479-2488. CrossRef Medline

Swann N, Poizner H, Houser M, Gould S, Greenhouse I, Cai W, Strunk J, George J, Aron AR (2011) Deep brain stimulation of the subthalamic nucleus alters the cortical profile of response inhibition in the beta frequency band: a scalp EEG study in Parkinson's disease. J Neurosci 31: 5721-5729. CrossRef Medline

van den Wildenberg WP, Burle B, Vidal F, van der Molen MW, Ridderinkhof KR, Hasbroucq T (2010) Mechanisms and dynamics of cortical motor inhibition in the stop signal paradigm: a TMS study. J Cogn Neurosci 22:225-239. CrossRef Medline

Verbruggen F, Logan GD (2009) Proactive adjustments of response strategies in the stop signal paradigm. J Exp Psychol Hum Percept Perform 35:835-854. CrossRef Medline

Verbruggen F, Chambers CD, Logan GD (2013) Fictitious inhibitory differences: how skewness and slowing distort the estimation of stopping latencies. Psychol Sci 24:352-362. CrossRef Medline

Vink M, Kahn RS, Raemaekers M, van den Heuvel M, Boersma M, Ramsey NF (2005) Function of striatum beyond inhibition and execution of motor responses. Hum Brain Mapp 25:336-344. CrossRef Medline

Wessel JR (2016) Testing multiple psychological processes for common neural mechanisms using EEG and independent component analysis. Brain Topogr 31(1):90-100. CrossRef

Wessel JR (2018) A neural mechanism for surprise-related interruptions of visuospatial working memory. Cereb Cortex 28:199-212. CrossRef Medline

Wessel JR (2017a) Perceptual surprise aides inhibitory motor control. J Exp Psychol Hum Percept Perform 43:1585-1593. CrossRef Medline

Wessel JR (2017b) Prepotent motor activity and inhibitory control demands in different variants of the go/no-go paradigm. Psychophysiology. In press. CrossRef Medline

Wessel JR, Aron AR (2013) Unexpected events induce motor slowing via a brain mechanism for action stopping with global suppressive effects. J Neurosci 33:18481-18491. CrossRef Medline

Wessel JR, Aron AR (2015) It's not too late: the onset of the frontocentral P3 indexes successful response inhibition in the stop signal paradigm. Psychophysiology 52:472-480. CrossRef Medline

Wessel JR, Aron AR (2017) On the globality of motor suppression: unexpected events and their influence on behavior and cognition. Neuron 93:259-280. CrossRef Medline

Wessel JR, Danielmeier C, Morton JB, Ullsperger M (2012) Surprise and error: common neuronal architecture for the processing of errors and novelty. J Neurosci 32:7528-7537. CrossRef Medline

Wessel JR, Reynoso HS, Aron AR (2013) Saccade suppression exerts global effects on the motor system. J Neurophysiol 110:883-890. CrossRef Medline

Wessel JR, Ullsperger M (2011) Selection of independent components representing event-related brain potentials: a data-driven approach for greater objectivity. Neuroimage, 54(3): 2105-2115. CrossRef 